Article

\title{
Particle-Hole Transformation in Strongly-Doped Iron-Based Superconductors
}

\author{
Jose P. Rodriguez \\ Department of Physics and Astronomy, California State University, Los Angeles, CA 90032, USA; \\ jrodrig@calstatela.edu
}

Received: 31 January 2019; Accepted: 7 March 2019; Published: 19 March 2019

\begin{abstract}
An exact particle-hole transformation is discovered in a local-moment model for a single layer of heavily electron-doped FeSe. The model harbors hidden magnetic order between the iron $d_{x z}$ and $d_{y z}$ orbitals at the wavenumber $(\pi, \pi)$. It potentially is tied to the magnetic resonances about the very same Néel ordering vector that have been recently discovered in intercalated FeSe. Upon electron doping, the local-moment model successfully accounts for the electron-pocket Fermi surfaces observed experimentally at the corner of the two-iron Brillouin zone in electron-doped FeSe, as well as for isotropic Cooper pairs. Application of the particle-hole transformation predicts a surface-layer iron-based superconductor at strong hole doping that exhibits high $T_{\mathcal{c}}$, and that shows hole-type Fermi-surface pockets at the center of the two-iron Brillouin zone.
\end{abstract}

Keywords: pnictides; chalcogenides; pairing symmetries; electronic structure

\section{Introduction}

The discovery of iron-based superconductors has identified a new route in the search for high critical temperatures [1]. Iron atoms in these materials lie in weakly coupled stacks of square lattices [2]. Electronic conduction resides within such layers, where charge carriers are primarily electrons/holes from iron $3 d$ levels. The optimum critical temperature in iron-pnictide materials, in particular, coincides with imperfect nesting between hole Fermi-surface pockets at the center of the Brillouin zone and electron Fermi-surface pockets at momenta along the principal axes of the square lattice of iron atoms that coincide with commensurate spin-density wave (cSDW) order. Strong hole doping can destroy such nesting. In particular, angle-resolved photoemission spectroscopy (ARPES) finds that the electron bands at cSDW momenta rise completely above the Fermi level in the series of compounds $\left(\mathrm{Ba}_{1-x} \mathrm{~K}_{x}\right) \mathrm{Fe}_{2} \mathrm{As}_{2}$ at $0.5<x<0.7$ [3]. ARPES on the end-member of the series $\mathrm{KFe}_{2} \mathrm{As}_{2}$, with superconducting $T_{\mathcal{C}} \cong 4 \mathrm{~K}$, reveals only hole Fermi surface pockets [4]. Density-functional-theory calculations recover the Lifshitz transtion at which the electron-type Fermi surface pockets disappear, but at a larger critical concentration of hole doping [5], $x_{c}=0.9$.

Strong electron doping can also destroy nesting in iron-based superconductors. ARPES on a monolayer of FeSe over a doped $\mathrm{SrTiO}_{3}$ (STO) substrate and ARPES on intercalated FeSe find only electron Fermi surface pockets at CSDW momenta [6-8]. Hole bands at the center of the Brillouin zone lie buried below the Fermi level. Unlike heavily hole-doped compounds such as $\mathrm{KFe}_{2} \mathrm{As}_{2}$, however, the FeSe surface layer shows high critical temperatures, $T_{c} \sim 100 \mathrm{~K}$, for superconductivity [9]. In addition, ARPES [6-8] and scanning tunneling microscopy (STM) $[10,11]$ on such surface layers of FeSe find evidence for an isotropic gap over the electron Fermi surface pockets, with no nodes. Finally, a Mott insulator phase is reported nearby at low electron doping in single-layer FeSe/STO and in voltage-gate tuned thin films of FeSe [12,13]. In contrast to itinerant magnetism, which is driven by Fermi-surface nesting, and which has some success in describing superconductivity in iron-pnictide materials [14,15], 
the limit of strong on-site electron repulsion $[16,17]$ may then be a valid starting point to describe superconductivity in heavily electron-doped FeSe.

Below, we identify a particle-hole transformation for a local-moment description of a single layer in an iron-based superconductor [16-18] that includes the minimum $d_{x z}$ and $d_{y z}$ iron orbitals [19-21]. At half filling of electrons, a doped Mott insulator results in the limit of strong iron-site Coulomb repulsion [18]. Above half filling (electron doping), mean field and exact calculations based on a hidden half metal state predict electronic structure that is very similar to that shown by heavily electron-doped $\left(\right.$ high- $\left.T_{c}\right)$ surface layers of FeSe [22,23]. The exact calculations also predict isotropic Cooper pairs at the electron Fermi surface pockets, in addition to remnant isotropic Cooper pairs of opposite sign on buried hole bands. Application of the particle-hole transformation to a surface layer of FeSe predicts a surface-layer iron-based superconductor that is heavily hole-doped, and that exhibits high $T_{\mathcal{C}}$ [21].

\section{Local-Moment Hamiltonian}

Our starting point is a two-orbital $t$ - $J$ model over the square lattice, where intra-orbital on-site Coulomb repulsion is strong $[16,17,19,20,24,25]$ :

$$
\begin{aligned}
H= & \sum_{\langle i, j\rangle}\left[-\left(t_{1}^{\alpha, \beta} c_{i, \alpha, s}^{\dagger} c_{j, \beta, s}+\text { h.c. }\right)+J_{1}^{\alpha, \beta} \mathbf{S}_{i, \alpha} \cdot \mathbf{S}_{j, \beta}\right]+ \\
& \sum[\langle i, j\rangle\rangle \\
& \left.\sum_{i}\left(J_{0} \mathbf{S}_{i, d-}^{\alpha, \beta} c_{i, \alpha, s}^{\dagger} \boldsymbol{S}_{j, \beta, s}+\text { h.c. }\right)+J_{2}^{\alpha, \beta} \mathbf{S}_{i, \alpha} \cdot \mathbf{S}_{j, \beta}\right]+ \\
\hline & \left.U_{0}^{\prime} \bar{n}_{i, d+} \bar{n}_{i, d-}+\lim _{U_{0} \rightarrow \infty} U_{0} n_{i, \alpha, \uparrow} n_{i, \alpha, \downarrow}\right) .
\end{aligned}
$$

Above, $\mathbf{S}_{i, \alpha}$ is the spin operator that acts on spin $s_{0}=1 / 2$ states of $d-=d_{(x-i y) z}$ and $d+=$ $d_{(x+i y) z}$ orbitals $\alpha$ in iron atoms at site $i$. Repeated orbital and spin indices in Equation (1) are summed over. Nearest neighbor and next-nearest neighbor Heisenberg exchange across the respective links $\langle i, j\rangle$ and $\langle\langle i, j\rangle\rangle$ is controlled by the coupling constants $J_{1}^{\alpha, \beta}$ and $J_{2}^{\alpha, \beta}$. They are due primarily to super exchange [16]. Hopping of an electron in orbital $\alpha$ to an unoccupied neighboring orbital $\beta$ is controlled by the matrix elements $t_{1}^{\alpha, \beta}$ and $t_{2}^{\alpha, \beta}$. Finally, $J_{0}$ is a ferromagnetic exchange coupling constant that imposes Hund's Rule. The last term in Equation (1) suppresses double occupancy at a site-orbital, where $n_{i, \alpha, s}=c_{i, \alpha, s}^{\dagger} c_{i, \alpha, s}$ is the occupation operator for a spin-s electron in orbital $\alpha$ at site $i$. The next-to-last term in Equation (1) measures the energy cost, $U_{0}^{\prime}>0$, of a pair of holes at an iron site, whereas $\bar{n}_{i, \alpha}=1-\sum_{s} n_{i, \alpha, s}$ counts holes at site-orbitals below half filling. Observe that $\bar{n}_{i, \alpha}$ can be replaced by $-\bar{n}_{i, \alpha}$, which counts singlet pairs at site-orbitals above half filling. Finally, notice that the operation $d \pm \rightarrow e^{ \pm i \theta} d \pm$ is equivalent to a rotation of the orbitals by an angle $\theta$ about the $z$ axis. Spin and occupation operators remain invariant under it. Magnetism described by the two-orbital $t-J$ model in Equation (1) is hence isotropic, which suppresses orbital order and nematicity [26,27].

Because the spin- $1 / 2$ moments live on isotropic $d \pm$ orbitals, two isotropic nearest neighbor and next-nearest neighbor Heisenberg exchange coupling constants exist:

$$
J_{n}^{\|}=J_{n}^{d \pm, d \pm} \quad \text { and } \quad J_{n}^{\perp}=J_{n}^{d \pm, d \mp} \quad(n=1,2) .
$$

The isotropy of the $d \pm$ orbitals also implies intra-orbital hopping matrix elements that are isotropic and real: $t_{n}^{\|}=t_{n}^{d \pm, d \pm}$ for $n=1,2$. Finally, the reflection properties of the $d_{x z}$ and $d_{y z}$ orbitals also imply real inter-orbital hopping matrix elements between nearest neighbors, with $d$-wave

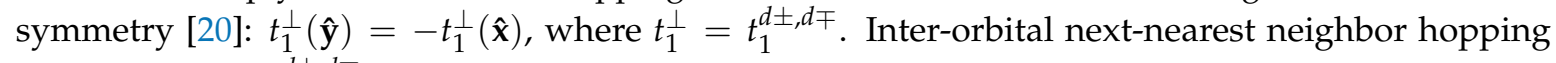
matrix elements $t_{2}^{d \pm, d \mp}$ also show $d$-wave symmetry, but they are pure imaginary. They consequently result in hybridization of the $d_{x z}$ and $d_{y z}$ orbital bands. Table 1 summarizes the expected phase diagram of the two-orbital $t-J$ model in Equation (1) near a quantum critical point into hidden magnetic order $[18,21,22]$. 
Table 1. Groundstate of two-orbital $t-J$ model in Equation (1) in the presence of magnetic frustration: $J_{2}^{\|}+J_{2}^{\perp}>\frac{1}{2}\left(J_{1}^{\|}+J_{1}^{\perp}\right)$. Hund coupling is tuned to the QCP at half filling, $\Delta_{c S D W} \rightarrow 0$ (Figure 1), which separates a cSDW when it's strong from hidden magnetic order when it is weak (Ref. [18]). Captions to Figures 1 and 2 give example model parameters.

\begin{tabular}{ccc}
\hline Filling, Bands & $J_{1}^{\|}<J_{1}^{\perp}$ & $J_{1}^{\|}>J_{1}^{\perp}$ \\
\hline half filling, none & hidden ferromagnet & hidden Néel \\
hole dope, hole bands @ $\Gamma$ & hidden half metal, FS @ $\Gamma$ & nested cSDW metal? \\
$e^{-}$dope, $e^{-}$bands @ M & nested cSDW metal? & hidden half metal, FS @ M \\
\hline
\end{tabular}

(A) HIDDEN SPINWAVE, $\mathrm{S}_{\mathrm{d}-}^{+}-\mathrm{S}_{\mathrm{d}+}^{+}$HIDDEN FERROMAGNET
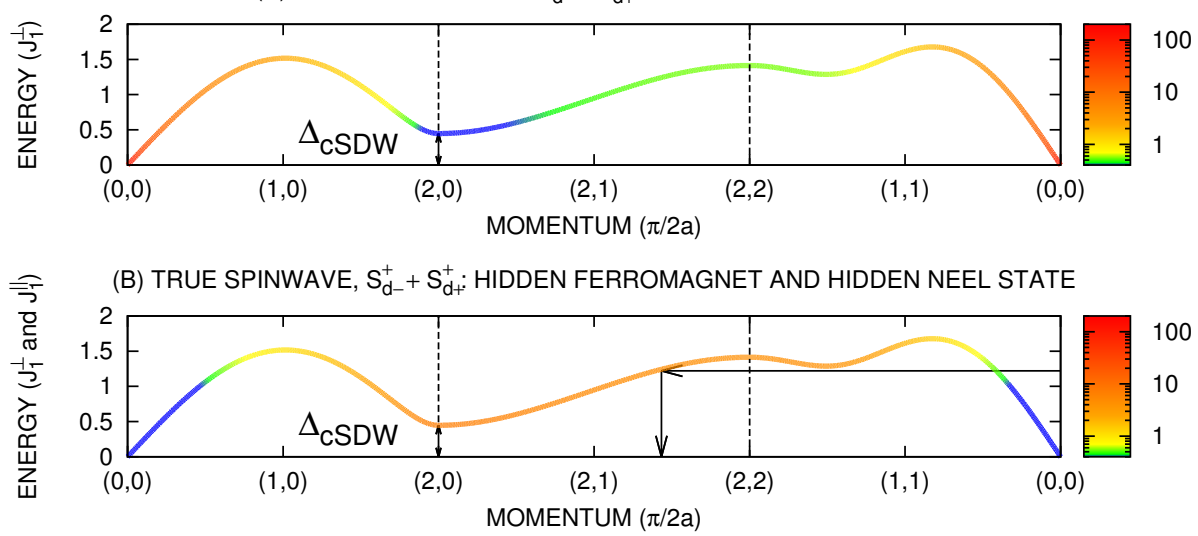

(C) HIDDEN SPINWAVE, $\mathrm{S}_{\mathrm{d}-}^{+}-\mathrm{S}_{\mathrm{d}+}^{+}$: HIDDEN NEEL STATE

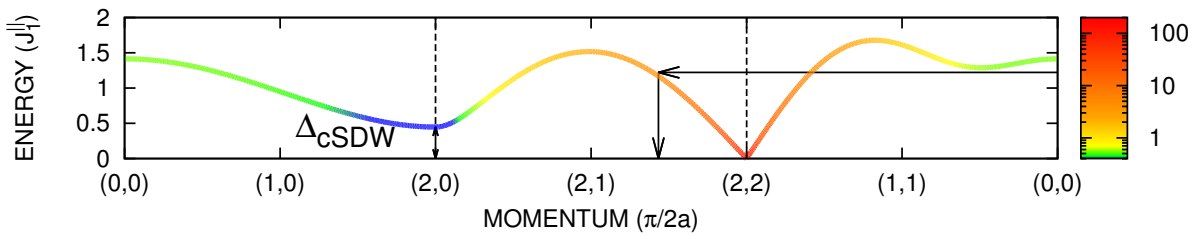

Figure 1. Linear spin-wave spectrum (Ref. [18]) for (A,B) hidden ferromagnet at Heisenberg coupling parameters $J_{1}^{\|}=0, J_{1}^{\perp}>0$, and $J_{2}^{\|}=0.3 J_{1}^{\perp}=J_{2}^{\perp}$, at Hund coupling $-J_{0}=-J_{0 c}-0.1 J_{1}^{\perp}$. Here, $-J_{0 c}$ is the critical Hund coupling at which $\Delta_{c S D W} \rightarrow 0$. Model parameters become (B,C) $J_{1}^{\|}>0, J_{1}^{\perp}=0$, $J_{2}^{\|}=0.3 J_{1}^{\|}=J_{2}^{\perp}$, and $-J_{0}=-J_{0 c}-0.1 J_{1}^{\|}$in the hidden Néel state after application of the particle-hole transformation. Color code represents spectral weight. 
(A) MOBILE HOLE AT $\mathrm{J}_{0}=0$

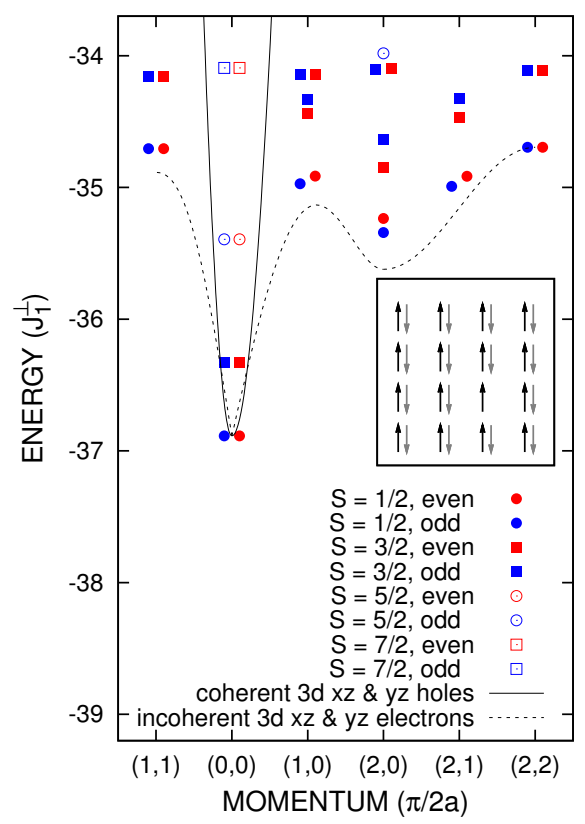

(B) MOBILE ELECTRON AT $\mathrm{J}_{0}=0$

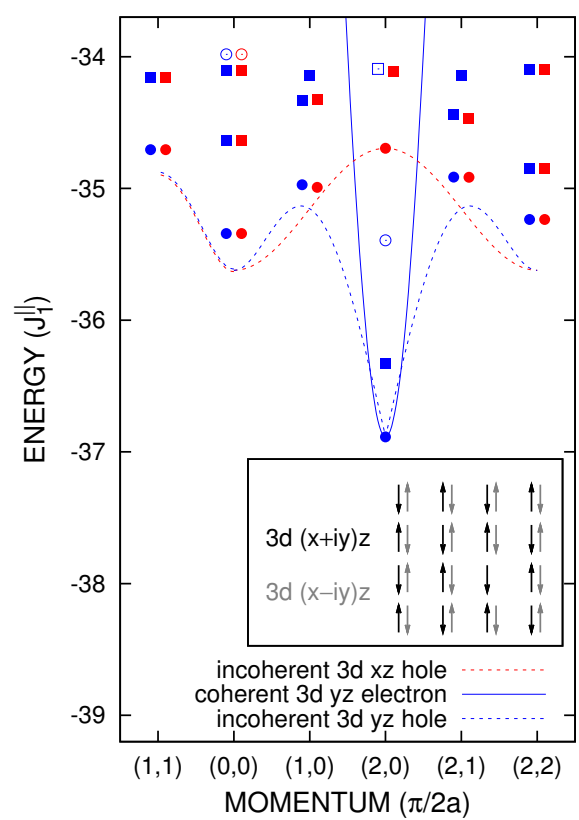

Figure 2. Exact spectra for $t-J$ model (Equation (1)), over a periodic $4 \times 4$ lattice, with hopping parameters (A) $t_{1}^{\|}=-3 J_{1}^{\perp}, t_{1}^{\perp}(\hat{\mathbf{x}})=-2 J_{1}^{\perp}, t_{1}^{\perp}(\hat{\mathbf{y}})=+2 J_{1}^{\perp}, t_{2}^{\|}=-J_{1}^{\perp}$, and $t_{2}^{d \pm, d \mp}=0$ in the mobile-hole case (31 electrons). Model parameters transform to $(\mathbf{B}) t_{1}^{\|}=2 J_{1}^{\|}, t_{1}^{\perp}(\hat{\mathbf{x}})=+3 J_{1}^{\|}, t_{1}^{\perp}(\hat{\mathbf{y}})=$ $-3 J_{1}^{\|}, t_{2}^{\|}=-J_{1}^{\|}$, and $t_{2}^{d \pm, d \mp}=0$ in the mobile-electron case (33 electrons). Heisenberg exchange coupling constants are given in the caption to Figure 1. Even/odd quantum number refers to parity under $P_{d, \bar{d}}$.

\section{Particle-Hole Transformation}

As shown below, the bipartite nature of the square lattice of iron atoms that stacks up to form iron-based superconductors allows us to define the following particle-hole transformation in momentum space for electrons in either the $d_{x z}$ or $d_{y z}$ orbitals. The corresponding electron destruction operator reads

$$
c_{S}\left(k_{0}, \mathbf{k}\right)=\mathcal{N}^{-1 / 2} \sum_{\alpha=0}^{1} \sum_{i} e^{-i\left(k_{0} \alpha+\mathbf{k} \cdot \mathbf{r}_{i}\right)} c_{i, \alpha, s},
$$

where $\mathcal{N}=2 N_{\mathrm{Fe}}$ denotes the number of sites-orbitals on the square lattice of iron atoms, and where the indices 0 and 1 denote the $d-$ and $d+$ orbitals $\alpha$. The quantum numbers $k_{0}=0$ and $\pi$ therefore represent the $d_{x z}$ and the $(-i) d_{y z}$ orbitals. We then define the particle-hole transformation by the replacements

$$
c_{S}\left(k_{0}, \mathbf{k}\right) \rightarrow c_{S}^{\dagger}\left(k_{0}, \mathbf{k}+\mathbf{Q}_{k_{0}}\right) \quad \text { and } \quad c_{S}^{\dagger}\left(k_{0}, \mathbf{k}\right) \rightarrow c_{S}\left(k_{0}, \mathbf{k}+\mathbf{Q}_{k_{0}}\right)
$$

where $\mathbf{Q}_{0}=(\pi / a) \hat{\mathbf{y}}$ and $\mathbf{Q}_{\pi}=(\pi / a) \hat{\mathbf{x}}$. Figure 3 displays the action of the above transformation on electronic structure: $(\mathrm{A}) \leftrightarrow(\mathrm{B})$. What then is the form of the above particle-hole transformation in real space for electrons in $d \pm$ orbitals? Comparison of Equations (2) and (3) yields the equivalent particle-hole transformation in real space:

$$
c_{i, \alpha, s} \rightarrow(-1)^{y_{i} / a} c_{i, p_{i}(\alpha), s}^{\dagger} \quad \text { and } \quad c_{i, \alpha, s}^{\dagger} \rightarrow(-1)^{y_{i} / a} c_{i, p_{i}(\alpha), s}
$$

where $p_{i}(d \pm)=d \pm$ for iron sites $i$ on the $A$ sublattice of the checkerboard, and where $p_{i}(d \pm)=d \mp$ for iron sites $i$ on the $B$ sublattice of the checkerboard. (see Appendix A for details.) 
It is useful now to note that application of the particle-hole transformation in Equation (4) results in a new next-nearest neighbor inter-orbital hopping matrix element $\bar{t}_{2}^{d \pm, d \mp}$ that is pure imaginary, but that alternates in sign between the $A$ versus the $B$ sites of the checkerboard. It does not describe mixing of the $d_{x z}$ and $d_{y z}$ orbitals in iron-based superconductors [20], and thus we turn it off entirely: $t_{2}^{d \pm, d \mp}=0$. The two-orbital $t$-J model Hamiltonian in Equation (1) now maintains its form after making the replacements Equation (4). Nearest neighbor model parameters, however, transform to

$$
\begin{array}{rll}
\bar{J}_{1}^{\|}=J_{1}^{\perp} & \text { and } & \bar{J}_{1}^{\perp}=J_{1}^{\|}, \\
\bar{t}_{1}^{\|}=-t_{1}^{\perp}(\hat{\mathbf{x}}) & \text { and } & \bar{t}_{1}^{\perp}(\hat{\mathbf{x}})=-t_{1}^{\|},
\end{array}
$$

with $\bar{t}_{1}^{\perp}(\hat{\mathbf{y}})=-\bar{t}_{1}^{\perp}(\hat{\mathbf{x}})$. Next-nearest neighbor model parameters $t_{2}^{\|}, J_{2}^{\|}$and $J_{2}^{\perp}$ remain unchanged. Finally, on-site parameters $J_{0}$ for ferromagnetic Hund coupling, $U_{0}$ for intra-orbital Coulomb repulsion, and $U_{0}^{\prime}$ for inter-orbital Coulomb repulsion also remain unchanged. Here, the occupation operators $n_{i, \alpha, s}$ in the divergent Hubbard term must be replaced by $1-n_{i, \alpha, s}$.

(A) HOLE DOPING
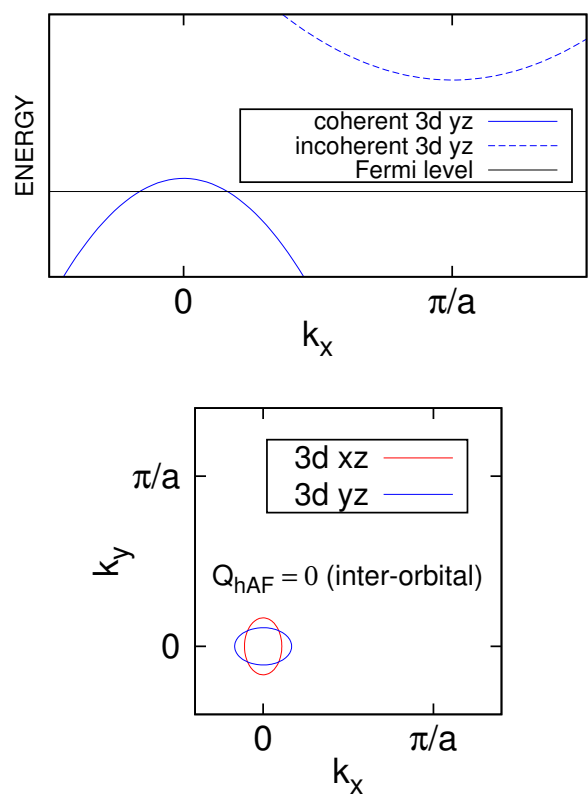

(B) ELECTRON DOPING
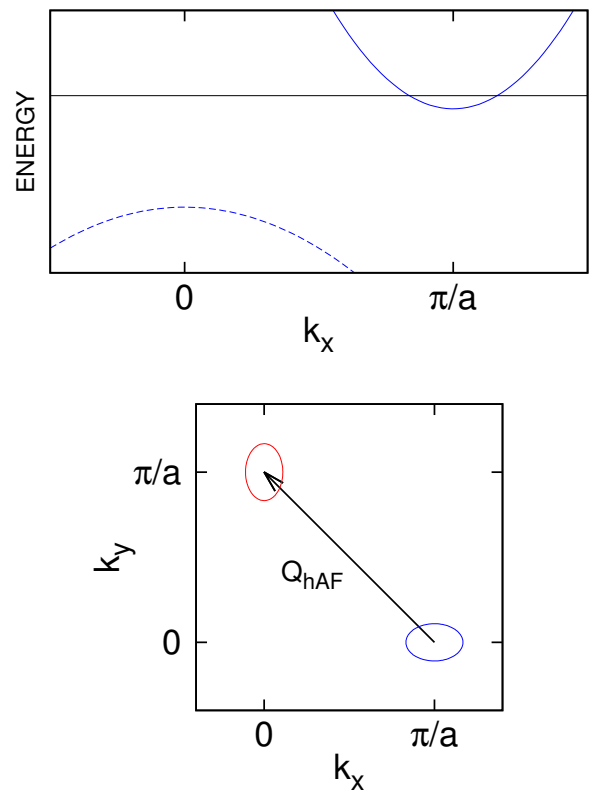

Figure 3. Electronic structure of half metal states characterized by hidden (inter-orbital) magnetic order at wavenumber $\mathbf{Q}_{\mathrm{hAF}}$ (see insets to Figure 2). Dispersions in energy are fixed at wavenumber $k_{y}=0$.

\section{Half Filling}

Consider half filling, with a density of electrons equivalent to one electron per site, per $d \pm$ orbital. No hopping of electron is then possible in the present limit, $U_{0} \rightarrow \infty$. It results in the Heisenberg model associated with the coupling constants $J_{0}, J_{n}^{\|}$and $J_{n}^{\perp}$ in the model Hamiltonian in Equation (1). The order parameter for hidden magnetic order at wavenumber $\mathbf{Q}$ is defined by $O_{h A F}=\sum_{i}\left\langle S_{i, d-}^{+}-S_{i, d+}^{+}\right\rangle \exp \left(i \mathbf{Q} \cdot \mathbf{r}_{i}\right)$, which is equal to

$$
O_{h A F}=\hbar \sum_{\mathbf{k}}\left\langle c_{\uparrow}^{\dagger}(0, \mathbf{k}+\mathbf{Q}) c_{\downarrow}(\pi, \mathbf{k})+c_{\uparrow}^{\dagger}(\pi, \mathbf{k}+\mathbf{Q}) c_{\downarrow}(0, \mathbf{k})\right\rangle
$$

Notice that such hidden magnetic order is equivalent to spin-density-wave order between the $d_{x z}$ and $d_{y z}$ orbitals [21,23]. Application of the particle-hole transformation in Equation (3) yields minus the complex conjugate of Equation (6) plus the replacement $\mathbf{Q} \rightarrow \mathbf{Q}+(\pi / a)(\hat{\mathbf{x}}+\hat{\mathbf{y}})$. In the presence of off-diagonal frustration, at weak enough Hund coupling, linear spin-wave theory applied to the 
resulting Heisenberg model in Equation (1) finds such long-range order at $\mathbf{Q}=0$ [18]. It corresponds to hidden ferromagnetic order [21]: $\nwarrow_{d-} \searrow_{d+}$ (see the inset to Figure 2A). Figure 1A,B shows the corresponding spin-wave spectra for the hidden ferromagnet at Heisenberg coupling constants $J_{1}^{\|}=0$, $J_{1}^{\perp}>0$, and $J_{2}^{\|}=0.3 J_{1}^{\perp}=J_{2}^{\perp}$, at sub-critical Hund coupling characterized by a spin gap $\Delta_{C S D W}>0$ at cSDW wavenumbers. The cSDW spin gap closes at a critical Hund's Rule coupling constant of $-J_{0 c}=2\left(J_{1}^{\perp}-J_{1}^{\|}\right)-4 J_{2}^{\|}$in such case $[18,19]$. Following the particle-hole transformation in Equation (5) of the model parameters, Figure 1B,C shows the spin-wave spectra for Heisenberg coupling constants $J_{1}^{\|}>0, J_{1}^{\perp}=0$, and $J_{2}^{\|}=0.3 J_{1}^{\|}=J_{2}^{\perp}$, but with $J_{0}$ unchanged. (The critical Hund's Rule coupling constant is now $-J_{0 c}=2\left(J_{1}^{\|}-J_{1}^{\perp}\right)-4 J_{2}^{\|}$.) Figure $1 \mathrm{C}$ displays a Goldstone mode at wavenumber $\mathbf{Q}=(\pi / a)(\hat{\mathbf{x}}+\hat{\mathbf{y}})$, which is evidence for a hidden Néel state. This hidden antiferromagnet shows opposing Néel order per $d \pm$ orbital (see the inset to Figure 2B and Ref. [22]), which is consistent with the particle-hole transformation in Equation (4) of the hidden ferromagnet. Notice that the spectrum of hidden spin-waves (Figure 1C) is obtained by shifting the spectrum of its particle-hole conjugate (Figure 1A) by the wavenumber $(\pi / a)(\hat{\mathbf{x}}+\hat{\mathbf{y}})[18]$.

True spin-wave and hidden spin-wave excitations, $\mathbf{S}_{d-}+\mathbf{S}_{d+}$ and $\mathbf{S}_{d_{-}}-\mathbf{S}_{d_{+}}$, are, respectively, even and odd under orbital swap, $P_{d, \bar{d}}$. Turning on hopping of electrons $t_{2}^{d \pm, d \mp}$ in Equation (1) that is pure imaginary, with $d$-wave symmetry, hybridizes the $d_{x z}$ and $d_{y z}$ orbitals [20], which breaks this symmetry away from half filling. It will mix true and hidden spin-waves, especially when they are degenerate. The arrows in Figure $1 B, C$ for spectra in the hidden Néel state show such degeneracy at four wavenumbers surrounding $\mathbf{Q}=(\pi / a)(\hat{\mathbf{x}}+\hat{\mathbf{y}})$ along the principal axes. Spin resonances in superconducting FeSe intercalates have been observed recently at these wavenumbers by inelastic neutron scattering [28-30]. This suggests that hidden Néel order is present in heavily electron-doped FeSe.

\section{One-Electron/One-Hole Bands}

We now compare spectra for one mobile hole and for one mobile electron with respect to half filling, with $t-J$ model parameters that are related to each other by the previous particle-hole transformation in Equation (5). In the hole-doped case, the Heisenberg exchange coupling constants coincide with the previous set for the hidden ferromagnet (Figure 1A), while the hopping matrix elements are set to $t_{1}^{\|}=-3 J_{1}^{\perp}, t_{1}^{\perp}(\hat{\mathbf{x}})=-2 J_{1}^{\perp}, t_{1}^{\perp}(\hat{\mathbf{y}})=+2 J_{1}^{\perp}, t_{2}^{\|}=-J_{1}^{\perp}$ and $t_{2}^{d \pm, d \mp}=0$. The latter implies that the $d_{x z}$ (even) versus the $d_{y z}$ (odd) orbital is a good quantum number. In the electron-doped case, nearest neighbor $t-J$ model parameters are set by Equation (5), while on-site and next-nearest neighbor model parameters are unchanged. Figure 2 shows exact spectra for one mobile hole and for one mobile electron roaming over a periodic $4 \times 4$ lattice of iron atoms, in the absence of Hund's Rule, $J_{0}=0$. The Schwinger-boson-slave-fermion representation of the correlated electron (hole) in the limit $U_{0} \rightarrow \infty$ was exploited in such case [31,32]. Details of the numerical calculation are given in the Supplementary Materials and in Ref. [20]. Notice that all of the states obey the particle-hole transformation in Equation (3). Figure 4 shows the one-electron spectra predicted by Schwinger-boson-slave-fermion mean field theory, but at $t_{1}^{\perp}=0$ for hole doping, and at $t_{1}^{\|}=0$ for electron doping. Notice that the states again obey the particle-hole relationship in Equation (3) depicted by Figure 3. 
(A) ONE HOLE: $t_{1}^{\|}=-3 J_{1}^{\perp}, t_{1}^{\perp}(x)=0=t_{1}^{\perp}(y), t_{2}^{\|}=-J_{1}^{\perp}, t_{2}^{\perp}(x+y)=0=t_{2}^{\perp}(y-x)$

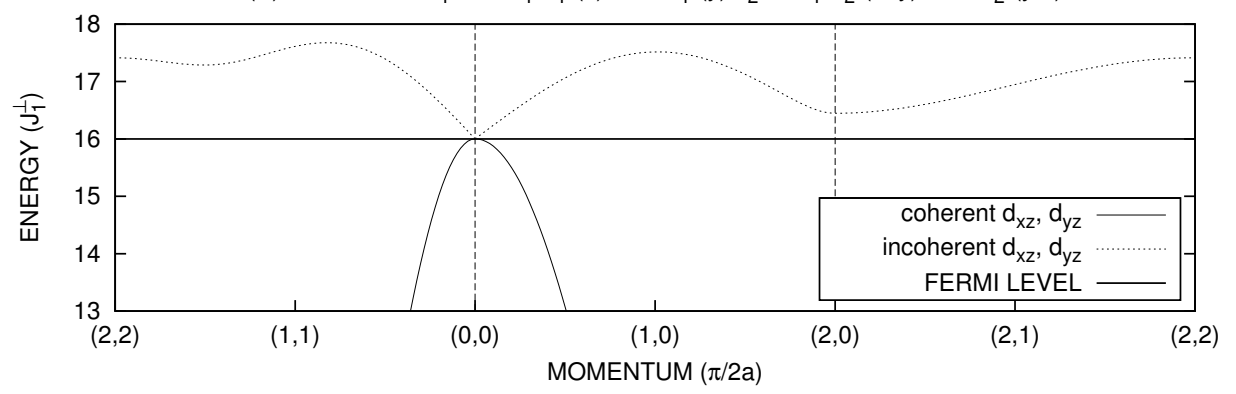

(B) ONE ELECTRON: $t_{1}^{\|}=0, t_{1}^{\perp}(x)=+3 J_{1}^{\|}, t_{1}^{\perp}(y)=-3 J_{1}^{\|}, t_{2}^{\|}=-J_{1}^{\|}, t_{2}^{\perp}(x+y)=0=t_{2}^{\perp}(y-x)$

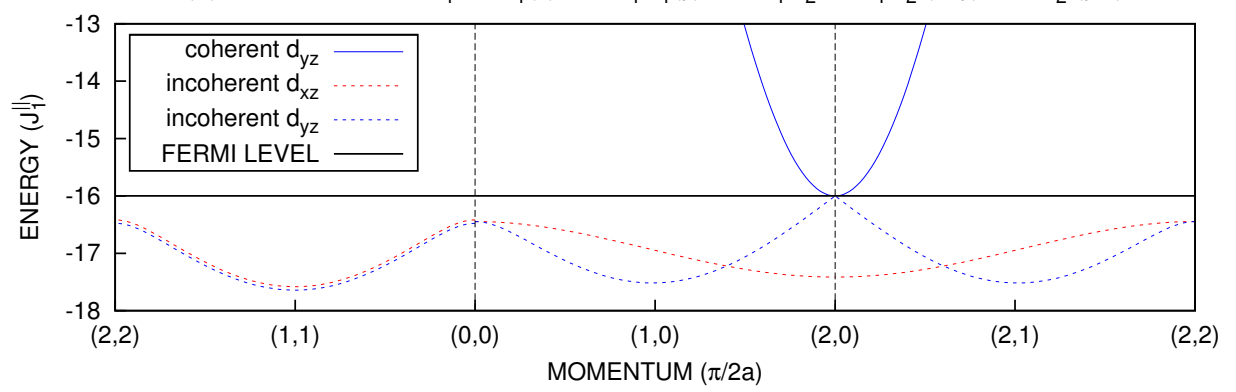

Figure 4. One-electron spectra of hidden half metal states within the mean field approximation at site-orbital concentration approaching half filling, $x \rightarrow 0$ (see refs. [21,22]). Heisenberg exchange coupling constants are set in the caption to Figure 1, while $J_{0}=J_{0 c}+0.1 J_{1}^{(\perp) \|}$.

The dispersion of the lowest-energy spin-1/2 mobile-hole states shown by Figure $2 \mathrm{~A}$ can be understood at ideal hopping, achieved by suppressing nearest-neighbor inter-orbital hopping as well: $t_{1}^{\perp} \rightarrow 0$. A half metal characterized by hidden magnetic order depicted by the inset to Figure $2 \mathrm{~A}$ is predicted in the absence of Hund's Rule at large electron spin $s_{0}[19,20]$. Electrons are spin polarized per $d \pm$ orbital, where they follow a hole-type energy dispersion relation $\varepsilon_{e}^{(0)}(\mathbf{k})=-2 t_{1}^{\|} \sum_{n=x, y} \cos k_{n} a-$ $2 t_{2}^{\|} \sum_{n=+,-} \cos k_{n} a$, with $k_{ \pm}=k_{x} \pm k_{y}$ (cf. the true half metal in ref. [33]). Two degenerate hole Fermi surface pockets at zero 2D moment are predicted for small concentrations of mobile holes per orbital, $x$, each with a Fermi wavenumber $k_{F} a=(4 \pi x)^{1 / 2}$ (see Figure 3A). The top of the hole-type band lies $\epsilon_{F}=\left|t_{1}^{\|}+2 t_{2}^{\|}\right|\left(k_{F} a\right)^{2}$ above the Fermi level. These coherent hole bands are recovered by a calculation of the one-electron propagator within a Schwinger-boson-slave-fermion mean-field approximation [31,32] of the two-orbital $t$-J model in Equation (1) for the above hidden half metal $[19,20]$. In the limit near half filling, at $|t| \gg J$, the one-electron propagator also reveals composite electron-spin-wave states at an energy $\epsilon_{F}+\hbar \omega_{\text {sw }}(\mathbf{k})$ above the Fermi level, where $\omega_{\mathrm{sw}}(\mathbf{k})$ is the spin-wave dispersion at large electron spin $s_{0}$ shown by Figure 1A,B [18] (see also Figure 4A). They are incoherent excitations that show intrinsic broadening [21]. The predicted dispersion relation is traced by the dashed line in Figure 2A in the absence of Hund's Rule. It compares well with the exact dispersion of the lowest-energy spin- $1 / 2$ excitations at non-ideal hopping matrix elements, in the absence of Hund's Rule, and it notably shows electron-type dispersion in the vicinity of cSDW wavenumbers $(\pi / a) \hat{\mathbf{x}}$ and $(\pi / a) \hat{\mathbf{y}}$. The latter are pulled down to lower energy as Hund coupling is turned on (cf. Figure S2a in Supplementary Materials). We therefore interpret the dispersion of those spin-1/2 groundstates, which, respectively, have odd and even parity under orbital swap $P_{d, \bar{d}}$, as emergent $d_{y z}$ and $d_{x z}$ electron bands.

Application of the particle-hole transformation in Equation (4) yields a new hidden half metal state depicted by the inset to Figure $2 \mathrm{~B}$, where the missing spin- $1 / 2$ moment in the third row represents a spin singlet (cf. the true half metal in Ref. [33]). By Equation (5), it is governed by the two-orbital $t$ - $J$ model in Equation (1) at electron doping above half-filling, with Heisenberg coupling constants that coincide with the previous set for the hidden Néel state (Figure 1C), and with hopping parameters $t_{1}^{\|}=+2 J_{1}^{\|}, t_{1}^{\perp}(\hat{\mathbf{x}})=+3 J_{1}^{\|}, t_{1}^{\perp}(\hat{\mathbf{y}})=-3 J_{1}^{\|}, t_{2}^{\|}=-J_{1}^{\|}$, and $t_{2}^{d \pm, d \mp}=0$. As $t_{1}^{\|} \rightarrow 0$, 
Schwinger-boson-slave-fermion mean field theory applied to the new model predicts circular electron Fermi surface pockets at cSDW wavenumbers similar to Figure 3B. It also predicts emergent hole excitations that disperse according to the dashed lines in Figure 2B [22] (see also Figure 4B). Again, the exact spectrum compares well to mean field theory. The first and second excited spin- $1 / 2$ states in Figure $2 \mathrm{~B}$ that lie at momentum zero and $(\pi / a)(\hat{\mathbf{x}}+\hat{\mathbf{y}})$ thereby correspond to a hole band plus its replica at lower energy, both buried below the Fermi level at zero 2D momentum in the two-iron folded Brillouin zone. Turning on Hund coupling pulls the first excited state at zero 2D momentum down in energy (cf. Figure S2b in Supplementary Materials). The previous prediction is consistent with reported evidence for such a replica band at the $\Gamma$ point from ARPES on FeSe/STO [7].

\section{Cooper Pairs with Emergent Sign Changes}

Consider now two electrons above half filling that roam over a $4 \times 4$ periodic lattice of iron atoms governed by the two-orbital $t-J$ model in Equation (1) [22]. Heisenberg exchange parameters are set to those listed in the caption to Figure $1 \mathrm{C}$, but new hopping matrix elements are chosen that leave the electron masses $m_{x}$ and $m_{y}$ per orbital unchanged at cSDW momenta: $t_{1}^{\|}=2 J_{1}^{\|}, t_{1}^{\perp}(\hat{\mathbf{x}})=+5 J_{1}^{\|}$, $t_{1}^{\perp}(\hat{\mathbf{y}})=-5 J_{1}^{\|}$, and $t_{2}^{\alpha, \beta}=0$. Such model parameters result in electron-type Fermi surface pockets centered at cSDW momenta in the hidden half metal state within the mean field approximation. Details of the exact calculation are given in the Supplementary Materials and in Ref. [21]. The Hund coupling, $-J_{0}$, is tuned to a putative quantum critical point (QCP) defined by degeneracy of the spin resonance at $\operatorname{cSDW}$ momenta with the hidden spin resonance at momentum $(\pi / a)(\hat{\mathbf{x}}+\hat{\mathbf{y}})$. This definition is suggested by the semi-classical analysis of the corresponding Heisenberg model at half filling (Figure 1C), which finds a QCP when the spin gap at cSDW momenta collapses [18]: $\Delta_{c S D W} \rightarrow 0$. A bound electron-pair groundstate exists below a continuum of states at zero net $2 \mathrm{D}$ momentum. It shows $S$-wave symmetry according to the reflection parities listed in Table 2 . An excited pair state with $D_{x^{2}-y^{2}}$ symmetry exists below the continuum as well.

Table 2. Reflection parities, orbital-swap parity, and spin of low-energy pair states with zero net momentum in order of increasing energy. The operator $R_{x^{\prime} z}$, for example, denotes a reflection about the $x^{\prime}-z$ plane, where $x^{\prime}$ is a diagonal axis. The hidden spin-wave in the case of electron doping is exceptional, where it carries net momentum $(\pi / a)(\hat{\mathbf{x}}+\hat{\mathbf{y}})$.

\begin{tabular}{cccccc}
\hline No. & Pair/Particle-Hole State & $\boldsymbol{R}_{x z}, \boldsymbol{R}_{\boldsymbol{y z}}$ & $\boldsymbol{R}_{x^{\prime} z}, \boldsymbol{R}_{\boldsymbol{y}^{\prime} \boldsymbol{z}}$ & $\boldsymbol{P}_{\boldsymbol{d}, \overline{\boldsymbol{d}}}$ & Spin \\
\hline 0 & $S$ & + & + & + & 0 \\
1 & $D_{x^{2}-y^{2}}$ & + & - & + & 0 \\
2 & hidden spin-wave & - & - & - & 1 \\
\hline
\end{tabular}

The order parameter for superconductivity is the defined as

$$
i F\left(k_{0}, \mathbf{k}\right)=\left\langle\Psi_{\text {Mott }}\left|\tilde{c}_{\uparrow}\left(k_{0}, \mathbf{k}\right) \tilde{c}_{\downarrow}\left(k_{0},-\mathbf{k}\right)\right| \Psi_{\text {Cooper }}\right\rangle
$$

times $\sqrt{2}$, where $\left|\Psi_{\text {Cooper }}\right\rangle$ is the groundstate of the electron pair, and where $\left\langle\Psi_{\text {Mott }}\right|$ is the groundstate of the Mott insulator at half filling. Above, the tilde notation signals the limit $U_{0} \rightarrow \infty$. Figure 5B depicts Equation (7) using exact groundstates $\left\langle\Psi_{\text {Mott }}\right|$ and $\left|\Psi_{\text {Cooper }}\right\rangle$ on a $4 \times 4$ periodic lattice of iron atoms at the putative QCP. In particular, the Hund coupling is tuned so that the groundstate spin-1 states at cSDW momenta, which have even parity under orbital swap, $P_{d, \bar{d}}$, become degenerate with the groundstate spin-1 state at wavenumber $(\pi / a)(\hat{\mathbf{x}}+\hat{\mathbf{y}})$, which has odd parity under orbital swap. The coupling constants, respectively, are $-J_{0}=1.35 J_{1}^{\|}$and $-J_{0}=2.25 J_{1}^{\|}$at half filling and for two mobile electrons. Notice that the order parameter displayed by Figure 5B is isotropic, but that it alternates in sign between the emergent hole bands at zero 2D momentum and the electron bands at cSDW momenta [22]. 
Figure 5A shows the particle-hole conjugate of the order parameter in Equation (7) for superconductivity in the two-orbital $t-J$ model with two-mobile holes that roam over a $4 \times 4$ periodic lattice, under the transformation in Equation (5) in parameter space [21]. Notice that it is related to Figure $5 \mathrm{~B}$ by the particle-hole transformation in Equation (3). In conclusion, both the electron pair and the conjugate hole pair display an $S^{+-}$order parameter for superconductivity, with remnant pairing on the emergent band of opposite sign. This result is similar to a recent proposal for $S^{+-}$pairing in heavily hole-doped iron superconductors that is based on a phenomenological attractive pairing interaction [34].

(A) TWO MOBILE HOLES

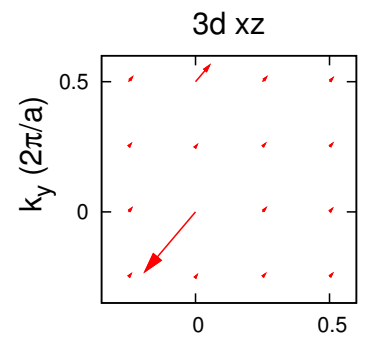

$3 d y z$

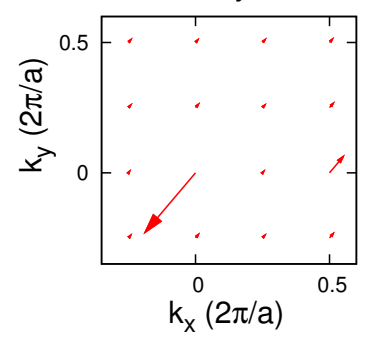

(B) TWO MOBILE ELECTRONS
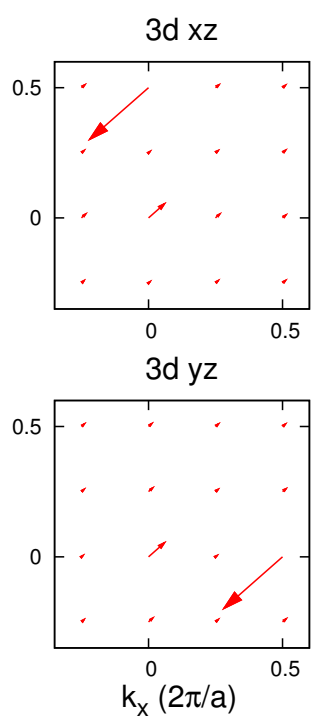

Figure 5. Complex order parameter for superconductivity (Equation (7)), with Heisenberg exchange coupling constants set in the caption to Figure 1 , and with hopping matrix elements (A) $t_{1}^{\|}=-5 J_{1}^{\perp}$, $t_{1}^{\perp}(\hat{\mathbf{x}})=-2 J_{1}^{\perp}, t_{1}^{\perp}(\hat{\mathbf{y}})=+2 J_{1}^{\perp}$, and $t_{2}^{\alpha, \beta}=0$ for two mobile holes (30 electrons). Nearest neighbor hopping matrix elements transform to $(\mathbf{B}) t_{1}^{\|}=2 J_{1}^{\|}, t_{1}^{\perp}(\hat{\mathbf{x}})=+5 J_{1}^{\|}$and $t_{1}^{\perp}(\hat{\mathbf{y}})=-5 J_{1}^{\|}$and $t_{2}^{\alpha, \beta}=0$ for two mobile electrons (34 electrons). In addition, inter-orbital on-site repulsion is set to $U_{0}^{\prime}=$ $\frac{1}{4} J_{0}+1000 J_{1}^{(\perp) \|}$, while the Hund coupling constant is set to $-J_{0}=2.25 J_{1}^{(\perp) \|}$. Heisenberg-exchange interactions in the Hamiltonian in Equation (1) are replaced with 1/2 the corresponding spin-exchange operators to reduce finite-size effects.

\section{Discussion and Conclusions}

Heavily electron-doped surface layers of FeSe show record superconducting critical temperatures as high as $T_{c} \cong 100 \mathrm{~K}$ [9]. ARPES reveals two electron Fermi-surface pockets at the corner of the two-iron Brillouin zone that cross, and that do not show level repulsion [6]. The electronic structure at the surface layer of heavily electron-doped FeSe can be described by the two-orbital $t-J$ model in Equation (1) at sub-critical Hund coupling, with hopping matrix elements and Heisenberg exchange coupling constants that favor the half metal state shown in the inset to Figure 2B. In particular, exact results and Schwinger-boson-slave-fermion mean field theory predict electron Fermi surface pockets centered at the two distinct cSDW momenta following Figure 3B (see Figure S2b in Supplementary Materials and Figure 4B). The Cooper pairs in heavily electron-doped FeSe surface layers are isotropic at the electron pockets [6-8], but we propose that they change sign at the buried hole bands according to Figure 5B [22].

Application of the particle-hole transformation in Equation (4) to the two-orbital $t-J$ model for a surface layer of FeSe implies a high- $T_{\mathcal{c}}$ surface layer at heavy hole doping that shows hole-type Fermi surface pockets at the center of the Brillouin zone (Figure $3 \mathrm{~A}$ ). It suggests searching for high- $T_{\mathcal{C}}$ superconductivity in surface layers of hole-doped iron-based compounds. 
Finally, from a purely technical perspective, the particle-hole transformation in Equation (4) of the two-orbital Hubbard model in Equation (1) for iron-based superconductors is a valuable tool that helps map out the parameter space. Figures 2 and 4 explicitly confirm the validity of the particle-hole transformation in the case of exact diagonalization on finite clusters and in the case of the mean-field approximation of the Schwinger-boson-slave-fermion formulation. The particle-hole transformation in Equation (4) will play a useful role in future analyses of the two-orbital Hubbard model in Equation (1) for iron-based superconductors by other techniques, such as by quantum Monte Carlo [35], and by experimental simulations using trapped atoms [36] and superconducting qubits [37].

Supplementary Materials: The following are available at http:/ /www.mdpi.com/2073-8994/11/3/396/s1.

Funding: This work was supported in part by the US Air Force Office of Scientific Research under grant Nos. FA9550-13-1-0118 and FA9550-17-1-0312, and by the National Science Foundation under PREM grant No. DMR-1523588.

Acknowledgments: The author thanks Miguel Araujo for correspondence. He also thanks Brent Andersen, Richard Roberts and Timothy Sell for technical help with the use of the virtual shared-memory cluster (Lancer) at the AFRL DoD Supercomputing Resource Center.

Conflicts of Interest: The author declare no conflict of interest.

\section{Appendix A. Two-Orbital Particle-Hole Transformation}

Below, we derive the particle-hole transformation for iron superconductors formulated in momentum space (Figure 3) and Equation (3)), starting from the transformation in real space in Equation (4):

$$
c_{i, \alpha, s} \rightarrow(-1)^{y_{i} / a} c_{i, p_{i}(\alpha), s}^{\dagger} \text { and } c_{i, \alpha, s}^{\dagger} \rightarrow(-1)^{y_{i} / a} c_{i, p_{i}(\alpha), s}
$$

where $p_{i}(d \pm)=d \pm$ for iron sites $i$ on the $A$ sublattice of the checkerboard, and where $p_{i}(d \pm)=d \mp$ for iron sites $i$ on the $B$ sublattice of the checkerboard. The creation operator for a spin $s$ electron that carries 3-momentum $\left(k_{0}, \mathbf{k}\right)$ is

$$
c_{S}^{\dagger}\left(k_{0}, \mathbf{k}\right)=\mathcal{N}^{-1 / 2} \sum_{\alpha=0}^{1} \sum_{i} e^{i\left(k_{0} \alpha+\mathbf{k} \cdot \mathbf{r}_{i}\right)} c_{i, \alpha, S^{\prime}}^{\dagger}
$$

where $\mathcal{N}=2 N_{\mathrm{Fe}}$ denotes the number of sites-orbitals on the square lattice of iron atoms, and where the indices 0 and 1 denote the $d-$ and $d+$ orbitals $\alpha$, respectively. The quantum numbers $k_{0}=0$ and $\pi$ therefore represent the $d_{x z}$ and the $(-i) d_{y z}$ orbitals.

Following Equation (A2), taking the Fourier transform of the first particle-hole transformation in Equation (A1) therefore yields that the destruction operator $c_{s}\left(k_{0}, \mathbf{k}\right)$ transforms to

$$
\mathcal{N}^{-1 / 2} \sum_{\alpha=0}^{1} \sum_{i} e^{i\left(k_{0} \alpha+\mathbf{k} \cdot \mathbf{r}_{i}\right)}(-1)^{y_{i} / a} c_{i, p_{i}(\alpha), s}^{\dagger}=\mathcal{N}^{-1 / 2} \sum_{\alpha=0}^{1} \sum_{i} e^{i\left[k_{0} \alpha+\left(\mathbf{k}+\frac{\pi}{a} \hat{\mathbf{y}}\right) \cdot \mathbf{r}_{i}\right]} c_{i, p_{i}(\alpha), s^{\prime}}^{\dagger}
$$

which is explicitly

$$
\mathcal{N}^{-1 / 2} \sum_{\alpha=0}^{1}\left\{\sum_{i \in A} e^{i\left(k_{0} \alpha+\left(\mathbf{k}+\frac{\pi}{a} \hat{\mathbf{y}}\right) \cdot \mathbf{r}_{i}\right)} c_{i, \alpha, s}^{\dagger}+\sum_{i \in B} e^{i\left[k_{0}(\alpha+1)+\left(\mathbf{k}+\frac{\pi}{a} \hat{\mathbf{y}}\right) \cdot \mathbf{r}_{i}\right]} c_{i, \alpha, s}^{\dagger}\right\} .
$$

Comparison with Equation (A2) yields that the above coincides with $c_{s}^{\dagger}\left(k_{0}, \mathbf{k}+\mathbf{Q}_{k_{0}}\right)$ at $k_{0}=0$, where $\mathbf{Q}_{0}=(\pi / a) \hat{\mathbf{y}}$. At $k_{0}=\pi$, on the other hand, the extra factor of $e^{i k_{0}}=-1$ in the second term of Equation (A4) can be replaced by an overall factor of $e^{i \frac{\pi}{a}(\hat{\mathbf{x}}+\hat{\mathbf{y}}) \cdot \mathbf{r}_{i}}$. Comparison with Equation (A2) in turn yields that Equation (A4) coincides with $c_{S}^{\dagger}\left(k_{0}, \mathbf{k}+\mathbf{Q}_{k_{0}}\right)$ at $k_{0}=\pi$, where $\mathbf{Q}_{\pi}=(\pi / a) \hat{\mathbf{x}}$. We thereby obtain the particle-hole transformation in momentum space: $c_{s}\left(k_{0}, \mathbf{k}\right) \rightarrow c_{s}^{\dagger}\left(k_{0}, \mathbf{k}+\mathbf{Q}_{k_{0}}\right)$. Using the identity $\mathbf{Q}_{k_{0}}+\mathbf{Q}_{k_{0}}=0$ that is true for crystal momentum yields the conjugate particle-hole transformation in momentum space: $c_{s}^{\dagger}\left(k_{0}, \mathbf{k}\right) \rightarrow c_{s}\left(k_{0}, \mathbf{k}+\mathbf{Q}_{k_{0}}\right)$. 


\section{References}

1. Kamihara, Y.; Watanabe, T.; Hirano, M.; Hosono, H. Iron-based layered superconductor La[O $\left.\mathrm{O}_{1-x} \mathrm{~F}_{x}\right] \mathrm{FeAs}$ $(x=0.05-0.12)$ with $T_{c}=26$ K. J. Am. Chem. Soc. 2008, 130, 3296-3297. [CrossRef] [PubMed]

2. Paglione, J.; Greene, R.L. High-temperature superconductivity in iron-based materials. Nat. Phys. 2010, 6, 645. [CrossRef]

3. Malaeb, W.; Shimojima, T.; Ishida, Y.; Okazaki, K.; Ota, Y.; Ohgushi, K.; Kihou, K.; Saito, T.; Lee, C.H.; Ishida, S.; et al. Abrupt change in the energy gap of superconducting $\mathrm{Ba}_{1-x} \mathrm{~K}_{x} \mathrm{Fe}_{2} \mathrm{As}_{2}$ single crystals with hole doping. Phys. Rev. B 2012, 86, 165117. [CrossRef]

4. Sato, T.; Nakayama, K.; Sekiba, Y.; Richard, P.; Xu, Y.-M.; Souma, S.; Takahashi, T.; Chen, G.F.; Luo, J.L.; Wang, N.L.; et al. Band structure and Fermi surface of an extremely overdoped iron-based superconductor $\mathrm{KFe}_{2} \mathrm{As}_{2}$. Phys. Rev. Lett. 2009, 103, 047002. [CrossRef] [PubMed]

5. Khan, S.N.; Johnson, D.D. Lifshitz transition and chemical instabilities in $\mathrm{Ba}_{1-x} \mathrm{~K}_{x} \mathrm{Fe}_{2} \mathrm{As}_{2}$ superconductors. Phys. Rev. Lett. 2014, 112, 156401. [CrossRef] [PubMed]

6. Peng, R.; Shen, X.P.; Xie, X.; Xu, H.C.; Tan, S.Y.; Xia, M.; Zhang, T.; Cao, H.Y.; Gong, X.G.; Hu, J.P.; et al. Measurement of an enhanced superconducting phase and a pronounced anisotropy of the energy gap of a strained FeSe single layer in $\mathrm{FeSe} / \mathrm{Nb}$ : $\mathrm{SrTiO}_{3} / \mathrm{KTaO}_{3}$ heterostructures using photoemission spectroscopy. Phys. Rev. Lett. 2014, 112, 107001. [CrossRef]

7. Lee, J.J.; Schmitt, F.T.; Moore, R.G.; Johnston, S.; Cui, Y.-T.; Li, W.; Yi, M.; Liu, Z.K.; Hashimoto, M.; Zhang, Y.; et al. Interfacial mode coupling as the origin of the enhancement of $T_{\mathcal{C}}$ in $\mathrm{FeSe}$ films on $\mathrm{SrTiO}_{3}$. Nature 2014, 515, 245. [CrossRef]

8. Zhao, L.; Liang, A.; Yuan, D.; Hu, Y.; Liu, D.; Huang, J.; He, S.; Shen, B.; Xu, Y.; Liu, X.; et al. Common electronic origin of superconductivity in $(\mathrm{Li}, \mathrm{Fe}) \mathrm{OHFeSe}$ bulk superconductor and single-layer FeSe $/ \mathrm{SrTiO}_{3}$ films. Nat. Commun. 2016, 7, 10608. [CrossRef]

9. Ge, J.-F.; Liu, Z.-L.; Liu, C.; Gao, C.-L.; Qian, D.; Xue, Q.-K.; Liu, Y.; Jia, J.-F. Superconductivity above $100 \mathrm{~K}$ in single-layer FeSe films on doped $\mathrm{SrTiO}_{3}$. Nat. Mater. 2015, 14, 285. [CrossRef]

10. Fan, Q.; Zhang, W.H.; Liu, X.; Yan, Y.J.; Ren, M.Q.; Peng, R.; Xu, H.C.; Xie, B.P.; Hu, J.P.; Zhang, T.; et al. Plain s-wave superconductivity in single-layer $\mathrm{FeSe}$ on $\mathrm{SrTiO}_{3}$ probed by scanning tunnelling microscopy. Nat. Phys. 2015, 11, 946. [CrossRef]

11. Yan, Y.J.; Zhang, W.H.; Ren, M.Q.; Liu, X.; Lu, X.F.; Wang, N.Z.; Niu, X.H.; Fan, Q.; Miao, J.; Tao, R.; et al. Surface electronic structure and evidence of plain s-wave superconductivity in $\left(\mathrm{Li}_{0.8} \mathrm{Fe}_{0.2}\right) \mathrm{OHFeSe}$. Phys. Rev. B 2016, 94, 134502. [CrossRef]

12. He, J.; Liu, X.; Zhang, W.; Zhao, L.; Liu, D.; He, S.; Mou, D.; Li, F.; Tang, C.; Li, Z.; et al. Electronic evidence of an insulator-superconductor crossover in single-layer FeSe/SrTiO 3 films. Proc. Natl. Acad. Sci. USA 2014, 111, 18501. [CrossRef] [PubMed]

13. Hanzawa, K.; Sato, H.H.; Kamiya, T.; Hosono, H. Electric field-induced superconducting transition of insulating FeSe thin film at 35 K. Proc. Natl. Acad. Sci. USA 2016, 113, 3986-3990. [CrossRef]

14. Hirschfeld, P.J.; Korshunov, M.M.; Mazin, I.I. Gap symmetry and structure of Fe-based superconductors. Rep. Prog. Phys. 2011, 74, 124508. [CrossRef]

15. Chubukov, A. Iron-Based Superconductivity; Springer Series in Materials Science: Berlin, Germany, 2015; Volume 211, p. 255.

16. Si, Q.; Abrahams, E. Strong correlations and magnetic frustration in the high $T_{\mathcal{C}}$ iron pnictides. Phys. Rev. Lett. 2008, 101, 076401. [CrossRef] [PubMed]

17. Rodriguez, J.P.; Rezayi, E.H. Low ordered magnetic moment by off-diagonal frustration in undoped parent compounds to iron-based high- $T_{c}$ superconductors. Phys. Rev. Lett. 2009, 103, 097204. [CrossRef] [PubMed]

18. Rodriguez, J.P. Magnetic excitations in ferropnictide materials controlled by a quantum critical point into hidden order. Phys. Rev. B 2010, 82, 014505. [CrossRef]

19. Rodriguez, J.P.; Araujo, M.A.N.; Sacramento, P.D. Fermi surfaces of iron pnictide high- $T_{C}$ superconductors from the limit of local magnetic moments. Phys. Rev. B 2011, 84, 224504. [CrossRef]

20. Rodriguez, J.P.; Araujo, M.A.N.; Sacramento, P.D. Emergent nesting of the Fermi surface from local-moment description of iron-pnictide high- $T_{c}$ superconductors. Eur. Phys. J. B 2014, 87, 163. [CrossRef]

21. Rodriguez, J.P. Collective mode at Lifshitz transition in iron-pnictide superconductors. J. Phys. Condens. Matter 2016, 28, 375701. [CrossRef] [PubMed] 
22. Rodriguez, J.P. Isotropic Cooper pairs with emergent sign changes in a single-layer iron superconductor. Phys. Rev. B 2017, 95, 134511. [CrossRef]

23. Rodriguez, J.P.; Melendrez, R. Fermi surface pockets in electron-doped iron superconductor by Lifshitz transition. J. Phys. Commun. 2018, 2, 105011 [CrossRef]

24. Nicholson, A.; Ge, W.; Zhang, X.; Riera, J.; Daghofer, M.; Oles, A.M.; Martins, G.B.; Moreo, A.; Dagotto, E. Competing pairing symmetries in a generalized two-orbital model for the pnictide superconductors. Phys. Rev. Lett. 2011, 106, 217002. [CrossRef] [PubMed]

25. Nicholson, A.; Ge, W.; Riera, J.; Daghofer, M.; Moreo, A.; Dagotto, E. Pairing symmetries of a hole-doped extended two-orbital model for the pnictides. Phys. Rev. B 2012, 85, 024532. [CrossRef]

26. Xu, C.; Müller, M.; Sachdev, S. Ising and spin orders in the iron-based superconductors. Phys. Rev. B 2008, 78, 020501(R). [CrossRef]

27. Yoshizawa, M.; Simayi, S. Anomalous elastic behavior and its correlation with superconductivity in iron-based superconductor $\mathrm{Ba}\left(\mathrm{Fe}_{1-x} \mathrm{Co}_{x}\right)_{2} \mathrm{As}_{2}$. Mod. Phys. Lett. B 2012, 26, 1230011. [CrossRef]

28. Davies, N.R.; Rahn, M.C.; Walker, H.C.; Ewings, R.A.; Woodruff, D.N.; Clarke, S.J.; Boothroyd, A.T. Spin resonance in the superconducting state of $\mathrm{Li}_{1-x} \mathrm{Fe}_{x} \mathrm{ODFe}_{1-y} \mathrm{Se}$ observed by neutron spectroscopy. Phys. Rev. B 2016, 94, 144503. [CrossRef]

29. Pan, B.; Shen, Y.; Hu, D.; Feng, Y.; Park, J.T.; Christianson, A.D.; Wang, Q.; Hao, Y.; Wo, H.; Zhao, J. Structure of spin excitations in heavily electron-doped $\mathrm{Li}_{0.8} \mathrm{Fe}_{0.2} \mathrm{ODFeSe}$ superconductors. Nat. Commun. 2017, 8, 123. [CrossRef]

30. Ma, M.; Wang, L.; Bourges, P.; Sidis, Y.; Danilkin, S.; Li, Y. Low-energy spin excitations in $\left(\mathrm{Li}_{0.8} \mathrm{Fe}_{0.2}\right) \mathrm{ODFeSe}$ superconductor studied with inelastic neutron scattering. Phys. Rev. B 2017, 95, 100504(R). [CrossRef]

31. Kane, C.L.; Lee, P.A.; Read, N. Motion of a single hole in a quantum antiferromagnet. Phys. Rev. B 1989, 39, 6880. [CrossRef]

32. Auerbach, A.; Larson, B.E. Doped antiferromagnet: The instability of homogeneous magnetic phases. Phys. Rev. B 1991, 43, 7800. [CrossRef]

33. Ptok, A.; Kapcia, K.J.; Cichy, A.; Oleś, A.M.; Piekarz, P. Magnetic Lifshitz transition and its consequences in multi-band iron-based superconductors. Sci. Rep. 2017, 7, 41979. [CrossRef]

34. Bang, Y. A shadow gap in the over-doped $\left(\mathrm{Ba}_{1-x} \mathrm{~K}_{x}\right) \mathrm{Fe}_{2} \mathrm{As}_{2}$ compound. New J. Phys. 2014, 16, 023029. [CrossRef]

35. Hirsch, J.E. Two-dimensional Hubbard model: Numerical simulation study. Phys. Rev. B 1985, $31,4403$. [CrossRef]

36. Casanova, J.; Mezzacapo, A.; Lamata, L.; Solano, E. Quantum simulation of interacting fermion lattice models in trapped ions. Phys. Rev. Lett. 2012, 108, 190502. [CrossRef]

37. Barends, R.; Lamata, L.; Kelly, J.; Garcia-Alvarez, L.; Fowler, A.G.; Megrant, A.; Jeffrey, E.; White, T.C.; Sank, D.; Mutus, J.Y.; et al. Digital quantum simulation of fermionic models with a superconducting circuit. Nat. Commun. 2015, 6, 7654. [CrossRef]

(C) 2019 by the author. Licensee MDPI, Basel, Switzerland. This article is an open access article distributed under the terms and conditions of the Creative Commons Attribution (CC BY) license (http://creativecommons.org/licenses/by/4.0/). 